Tohoku J. exp. Med., 1969, 99, 25-33

\title{
Latent Autoimmune Hemolytic Syndrome
}

\author{
Takeo Kuroyanagi and Hajime Sugiyama
}

\author{
The Third Department of Internal Medicine (Prof. K. Nakao), \\ Faculty of Medicine, University of Tokyo, Tokyo
}

\begin{abstract}
The direct anti-globulin test was performed at random on 310 hospitalized patients with various diseases. Thirty-three patients were positive to anti-globulin test without any evidence of manifest autoimmune hemolytic anemia.

Hematologic and erythrokinetic studies on these patients with latent autoim. mune hemolytic syndrome revealed that their slight anemia was secondary anemia due to their basic diseases with a slightly increased hemolysis.

The basic diseases of these patients were autoimmune diseases, mesenchymal disease, chronic infections, hypersensitivity disease, pulmonary silicosis associated with tuberculosis, leukemia, Hodgkin's disease and others.

Anti-erythrocyte autoantibodies were of warm type and intermediate type except for one case of $\gamma$-globulin type. The immunoglobulin class of anti-erythrocyte autoantibodies was IgG. Their light chain type was as follows: $\kappa$ type, $2 ; \lambda$ type 5; and $k+\lambda$ type, 1 . The coexistence of other various autoantibodies was demonstrated in most of the patients.

A marked increase of peripheral large lymphocytes, hyperplasia of germinal centers associated with proliferations of reticulum cells and lymphogonias (immunoblasts) and a marked infiltration of plasma cells were demonstrated in biopsied lymph nodes.

The mechanism of the association of latent autoimmune hemolytic syndrome with basic diseases was discussed.
\end{abstract}

Hennemann ${ }^{1,2}$ has reported an acquired latent hemolytic state induced by anti-erythrocyte autoantibodies, in which no manifest sign or result of increased hemolysis is found in spite of positive direct anti-globulin test. He has classified the latent autoimmune hemolytic syndrome into symptomatic and idiopathic types.

Thirty-three patients with symptomatic latent autoimmune hemolytic syndrome were studied hematologically and immunohematologically.

\section{Methods}

The direct anti-globulin test was performed at random on 310 hospitalized patients with various diseases. The hemagglutination of direct anti-globulin test was evaluated microscopically. The indirect anti-globulin test was performed on patients with positive direct anti-globulin tests. Immunoglobulin classes and light chain type of anti-erythrocyte autoantibodies were determined with eluates from red blood cells of patients with latent autoimmune hemolytic syndrome by the indirect Coombs test using anti-IgG, anti-IgA, anti-IgM, anti-K and anti- $\wedge$ antibodies, respectively.

Received for publication, May 13, 1969. 
Red blood cell counts, hemoglobin contents, reticulocyte counts and myelograms were determined on patients with latent autoimmune hemolytic syndrome. Osmotic fragility of red blood cells and erythrokinetics were studied on several patients with the same syndrome.

Immunological abnormalities were studied on patients with latent autoimmune hemolytic syndrome. Antinuclear, anti-thyroid and anti-stomach auto-antibodies were examined by the immunofluorescence method. Autoimmune complement fixation test ${ }^{3}$ (AICF) was performed using liver homogenates of rats as antigens. Anti-platelet antibodies were measured by complement fixation test. Anti-leukocyte antibodies,6 were examined by leukoagglutination test. Rheumatoid factors were measured by Waaler-Rose reaction and RA-test.

Lymph node biopsy was performed on several patients with latent autoimmune hemolytic syndrome.

\section{RESULTS}

\section{1) Diseases associated with latent autoimmune hemolytic syndrome}

Thirty-three among 310 examined patients demonstrated positive direct antiglobulin test without any evidence of manifest autoimmune hemolytic anemia. Diseases of patients associated with latent autoimmune hemolytic syndrome are shown in Table 1.

2) Antibody type, immunoglobulin class and light chain type of antierythrocyte autoantibodies (Table 2)

The indirect anti-globulin test was performed on serum of 23 patients with positive direct anti-globulin test. It was positive in 14 out of 23 patients.

The antibody type of anti-erythrocyte autoantibodies was studied on serum of 13 patients with positive indirect antiglobulin test. All patients demonstrated warm type anti-erythrocyte autoantibodies. Intermediate type autoantibodies were demonstrated in 12 out of 13 patients and $\gamma$-globulin type autoantibodies in a patient.

Immunoglobulin classes were determined with eluates from red blood cells of 13 patients with latent autoimmune hemolytic syndrome by the indirect Coombs

TABLE 1. Diseases associated with latent autoimmune hemolytic syndrome Autoimmune diseases

Systemic lupus erythematosis

Myasthenia gravis

Dermatomyositis

Hashimoto's disease

Mesenchymal disease

Chronic infection \& hypersensitivity

Pulmonary silicosis associated with tuberculosis

Malignant tumor

Leukemia

Hodgkin's disease

Cancer

Thymoma

Miscellaneous

6

2

1

I

3

5

3

7

3

2

1

1 
TABLE 2. Characteristics of autoantibodies in latent autoimmune hemolytic syndrome

\begin{tabular}{|c|c|c|c|}
\hline & - & $\begin{array}{c}\text { Examined } \\
\text { cases }\end{array}$ & $\begin{array}{c}\text { Positive } \\
\text { cases }\end{array}$ \\
\hline \multirow{2}{*}{ Coombs test } & Direct & 23 & 23 \\
\hline & Indirect & 23 & 14 \\
\hline \multirow{3}{*}{ Antibody type } & Warm & 13 & 13 \\
\hline & Intermediate & 13 & 12 \\
\hline & $\gamma$-Globulin & 13 & 1 \\
\hline \multirow{3}{*}{ L-chain type } & $\mathbf{K}$ & 8 & 2 \\
\hline & L & 8 & 5 \\
\hline & $\mathbf{K}+\mathbf{L}$ & 8 & 1 \\
\hline
\end{tabular}

test using anti-IgG, anti-IgA and anti-IgM antibodies. All the patients showed IgG antierythrocyte autoantibodies.

Light chain type of autoantibodies was examined with eluates from red blood cells of 8 patients with latent autoimmune hemolytic syndrome. Two patients showed $\kappa$ type, 5 patients $\lambda$ type and a patient, $\kappa+\lambda$ type.

3) Hematologic examinations on patients with latent autoimmune hemolytic syndrome

Hemoglobin contents averaged $11.5 \mathrm{~g} / 100 \mathrm{ml}$, ranging from $62 . \mathrm{g} / 100 \mathrm{ml}$ to $15 \mathrm{~g} /$ $100 \mathrm{ml}$, as shown in Fig. 1. Red blood cell counts averaged $343 \times 10^{4} / \mathrm{cmm}$. Color indices in 22 patients were within normal limits. Two patients gave hypochromic color indices and two hyperchromic.

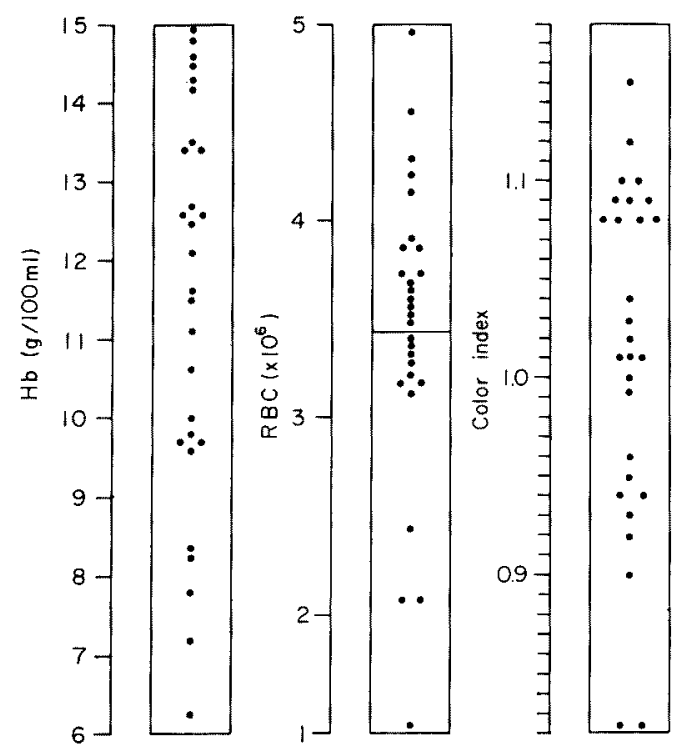

Fig. 1. Hb, RBC counts and color index in latent autoimmune hemolytic syndrome. 

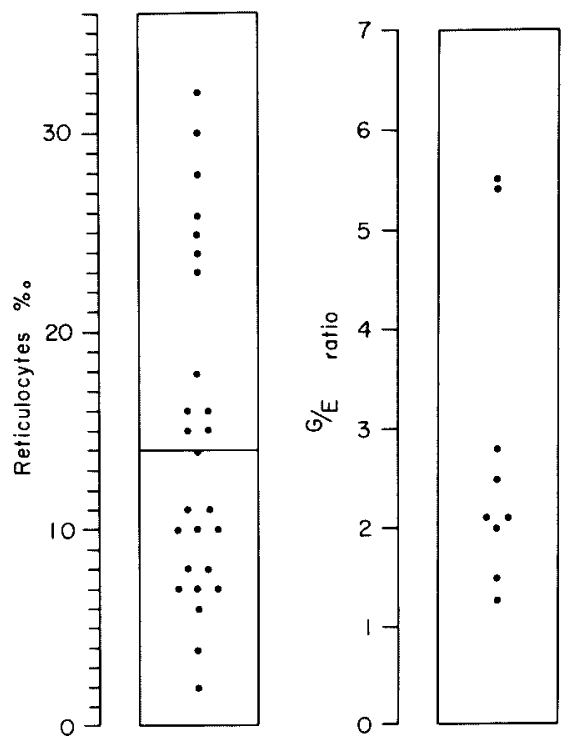

Fig. 2 Reticulocytes and $\mathrm{G} / \mathrm{E}$ ratio in latent autoimmune hemolytic syndrome.

Reticulocyte counts averaged $1.4 \%$, ranging from 0.2 to $3.2 \%$, as shown in Fig. 2. Twelve out of 26 patients showed a moderate reticulocytosis.

Myelograms were studied on ten patients with latent autoimmune hemolytic syndrome. The $\mathbf{G} / \mathbf{E}$ ratio was within normal limits.

Osmotic fragility of red blood cells and autohemolysis were measured on three patients with latent autoimmune hemolytic syndrome. All patients had normal osmotic fragility of red blood cells and normal autohemolysis test, as shown in Fig. 3.

Erythrokinetics were examined on three patients with latent autoimmune hemolytic syndrome (Table 3). Plasma iron content was $100 \mu \mathrm{g} / 100 \mathrm{ml}, 126 \mu \mathrm{g} /$ $100 \mathrm{ml}$ and $87 \mu \mathrm{g} / 100 \mathrm{ml}$. Half time of plasma iron disappearance (PID T/2) was 1.3 hours, 1.5 hours and 1.5 hours. Plasma iron turnover rate (PIT) was $35.3 \mathrm{mg} /$ day, $30.6 \mathrm{mg} /$ day and $27.8 \mathrm{mg} /$ day, indicating a slight increase. Red blood cell iron turnover rate (RIT) was $35.5 / \mathrm{mg} /$ day, $30.6 / \mathrm{mg} /$ day and $23.6 / \mathrm{mg} /$ day, demonstrating a moderate increase. Half time of red blood cell life span determined by the ${ }^{51} \mathrm{Cr}$ method was 16.2 days, 18.5 days and 20 days, demonstrating a moderate shortening of the life span. Hemolytic index was 35,32 and 28 , indicating a slight increase.

4) Immunological abnormalities in patients with latent autoimmune hemolytic syndrome

Erythrocyte sedimentation rate was accelerated in 24 out of 30 patients, as shown in Fig. 4. C-reactive protein was positive in $45 \%$ of the patients. Serum gammaglobulin was elevated in 20 out of 23 patients (Fig. 4).

Autoantibodies other than anti-erythrocyte autoantibodies were demonstrated in 22 out of 33 patients with latent autoimmune hemolytic syndrome (Table 4). 

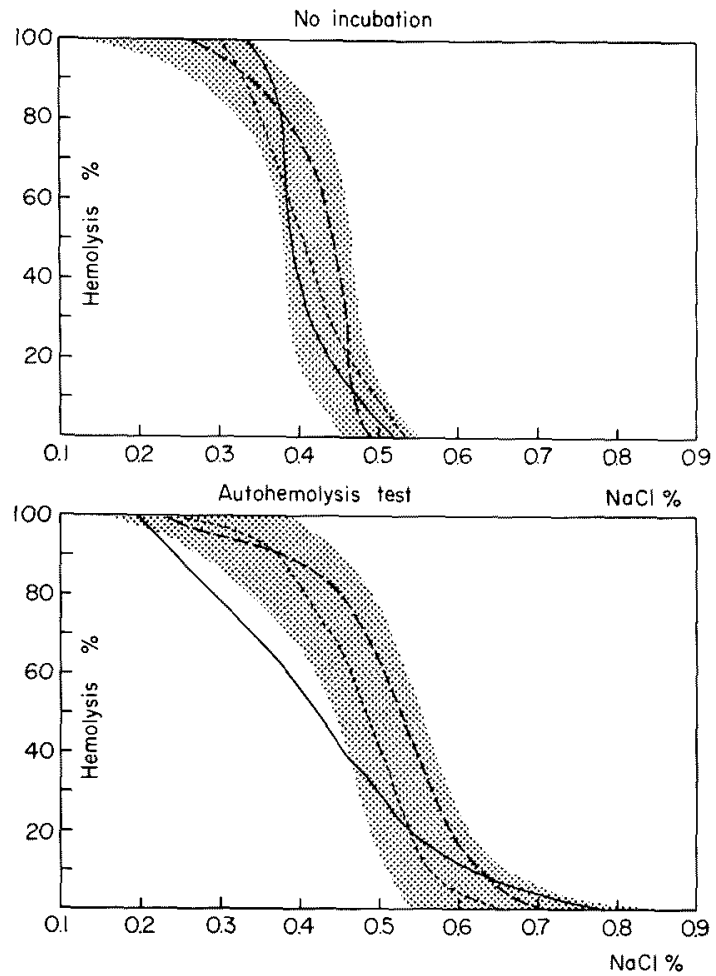

Fig. 3. Red blood cell osmotic fragility in latent autoimmune hemolytic syndrome. No incubation.

TABLE 3. Erythrokinetics in patients with latent autoimmune hemolytic syndrome

\begin{tabular}{|c|c|c|c|}
\hline & Y A & $\mathrm{OE}$ & $\mathrm{H} \mathrm{U}$ \\
\hline $\mathrm{Hb} \quad(\mathrm{g} / \mathrm{dl})$ & 13.2 & 11.6 & 12.2 \\
\hline $\operatorname{RBC} \quad\left(10^{4}\right)$ & 375 & 326 & 386 \\
\hline Ret $(\%)$ & 18 & 25 & 7 \\
\hline $\operatorname{SeFe}(\mu \mathrm{g} / \mathrm{dl})$ & 100 & 126 & 87 \\
\hline PID (hr) & 1.3 & 1.5 & 1.5 \\
\hline $\operatorname{PIT}(\mathrm{mg} / \mathrm{d})$ & 35.3 & 30.6 & 27.8 \\
\hline $\operatorname{RIT}(\mathrm{mg} / \mathrm{d})$ & 35.3 & 30.6 & 25.6 \\
\hline Life span & 16.2 & 18.5 & 20 \\
\hline $\mathrm{HI}$ & 35 & 32 & 28 \\
\hline
\end{tabular}

Note: HI, hemolytic index

Anti-nuclear autoantibodies were positive in 10 patients, anti-stomach autoantibodies in 6 patients, rheumatoid factors in 5 patients, AICF in 3 patients, anti-thyroid autoantibodies in 2 patients, anti-platelet antibodies in 2 patients. Two patients gave a biologically false postive Wasserman reaction. The PaulBunnel reaction was positive in two patients. 

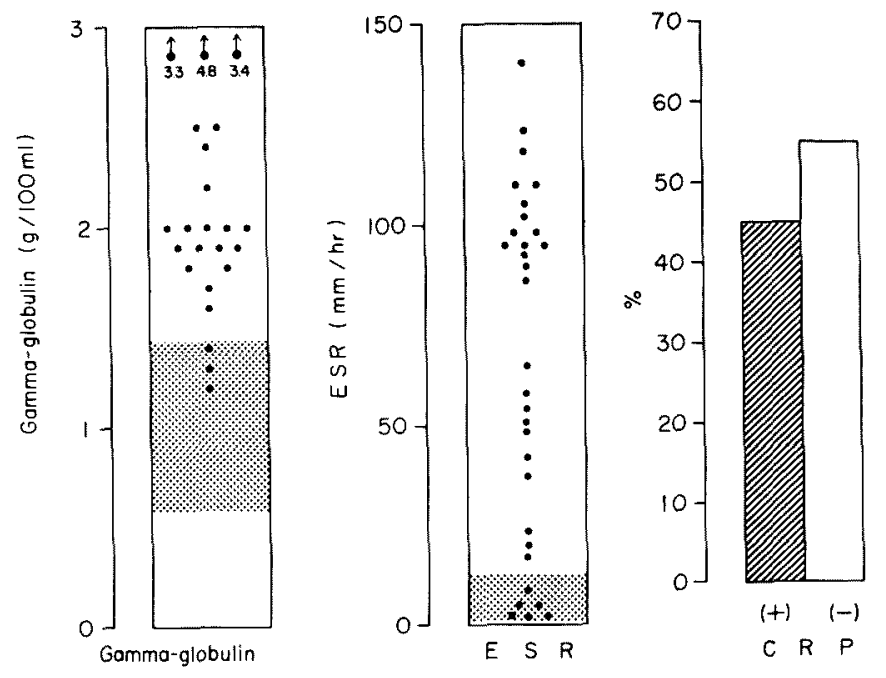

Fig. 4 Gammaglobulin, erythrocyte sedimentation rate and CRP in latent autoimmune hemolytic syndrome.

$\mathrm{T}_{\mathrm{ABLE}}$ 4. Autoimmune phenomenon in patients with latent autoimmune hemolytic syndrome

\begin{tabular}{l|c}
\hline Autontibodies Positive & $22 / 33$ \\
\hline Anti-nuclear & 10 \\
Anti-stomach & 6 \\
RA-Factor & 5 \\
AICF & 3 \\
Wa-R False positive & 3 \\
Anti-thyroid & 2 \\
Paul-Bunnell & 2 \\
Anti-platelet & 2 \\
Anti-leukocyte & 2
\end{tabular}

5) Histologic examination of biopsied lymph nodes of patients with latent autoimmune hemolytic syndrome

Lymphocyte counts were measured on patients with latent autoimmune hemolytic syndrome. Lymphocytosis above $3,000 / \mathrm{cmm}$ was found in only $9 \%$ of patients. However, an increase of large lymphocytes was demonstrated in $67 \%$ of patients (Table 5 ).

The lymph nodes biopsy was performed on three patients with mesenchymal disease and five patients with chronic infections and hypersensitivity disease. Histological examination of biopsied lymph nodes demonstrated a marked hyperplasia of germinal centers associated with proliferation of reticulum cells and lymphogonias (immunoblasts). Plasma cell infiltration was also evident. 
TABLE 5. Incidence of an increase of large lymphocytes in latent autoimmune hemolytic syndrome, systemic lupus erythematosus and myasthenia gravis

\begin{tabular}{c|c|c|c|c|c|c}
\hline & \multicolumn{2}{|c|}{ LAIH } & \multicolumn{2}{c|}{ SLE } & \multicolumn{2}{c}{ MG } \\
\hline Ly $3,500 \mathrm{cmm}$ & No. & $\%$ & No. & $\%$ & No. & $\%$ \\
\hline Ll $700 \mathrm{cmm}$ & 333 & 9.1 & 225 & 8.0 & $\mathbf{7 7 4}$ & 9.4 \\
\hline & 2233 & 66.7 & 2225 & 88.0 & 5069 & 72.5
\end{tabular}

Note: LAIH: latent autoimmune hemolytic syndrome. SLE: systemic lupus erythematosus. MG: myasthenia gravis. Ly: lymphocyte. Ll: large lymphocyte.

\section{Comments}

The direct anti-globulin test was performed at random on 310 hospitalized patients with various diseases. Thirty three patients gave a positive anti-globulin test without any evidence of manifest autoimmune hemolytic anemia. All the patients had some basic diseases. Therefore, it is evident that these patients were associated with symptomatic latent autoimmune hemolytic syndrome.

The basic diseases of these patients associated with latent autoimmune hemolytic syndrome were autoimmune diseases such as systemic lupus erythematosus (SLE), myasthenia gravis, dermatomyositis, Hashimoto's disease, chronic infections, hypersensitivity disease, mesenchymal disease, pulmonary silicosis with tuberculosis, leukemia, stomach cancer, Hodgkin's disease and others.

The indirect anti-globulin test was positive in 14 out of 23 patients with the positive direct anti-globulin test. All the anti-erythrocyte autoantibodies were warm type. The intermediate type autoantibodies were demonstrated in 12 out of 13 patients. Only one patient showed $\gamma$-globulin type autoantibodies. All the antierythrocyte autoantibodies were IgG. Light chain type of antierythrocyte autoantibodies was as follows: $\kappa$ type, 2 cases; $\lambda$ type, 5 cases; and $\kappa+\lambda$ type, one case. This distribution pattern of light chain type of anti-erythrocyte autoantibodies was similar to that in Japanese patients ${ }^{7}$ with autoimmune hemolytic anemia.

Most of patients with latent autoimmune hemolytic syndrome demonstrated a slight normochromic anemia and a slight reticulocytosis. They showed a slight increase of plasma iron turnover rate and red blood cell iron turnover rate. However, both the shortening of red blood cell life span and an increase of hemolytic index were too slight to attribute anemia of these patients to hemolysis. It seems likely that anemia in these patients might be a secondary one due to patients' basic diseases.

Patients associated with latent autoimmune hemolytic syndrome demonstrated the coexistence of various autoantibodies, indicating immunological abnormalities in their immunocyte system.

The latent autoimmune hemolytic syndrome in patients with autoimmune diseases such as SLE, myasthenia gravis, dermatomyositis and Hashimoto's disease may be probably due to the coproduction of anti-erythrocyte autoantibodies. 
Kuroyanagi et al..$^{8}$ have reported an increase of peripheral large lymphocytes in patients with autoimmune diseases such as SLE and myasthenia gravis, pointing out a close relationship between the increase of large lymphocytes and the hyperplasia of germinal centers in lymphatic tissues. Generally speaking, there is a marked germinal center hyperplasia associated with proliferations of reticulum cells and lymphogonias ${ }^{9}$ (immunoblasts ${ }^{10)}$ in patients with autoimmune diseases), suggesting that such changes in lymphatic tissues might reflect autoantibody production.

Patients associated with latent autoimmune hemolytic syndrome demonstrated a marked increase of peripheral large lymphocytes. The histological examination of biopsied lymph nodes of latent autoimmune hemolytic syndrome patients who were associated with mesenchymal disease, chronic infections and hypersensitivity disease, demonstrated a marked hyperplasia of germinal centers associated with proliferations of reticulum cells and lymphogonias (immunoblasts) and a marked increase of plasma cells. These results suggest that the association of latent autoimmune hemolytic syndrome in these patients is probably due to immunological aberrations of their immunocyte system.

Kamijima ${ }^{11}$ has reported that patients with pulmonary silicosis demonstrate frequently the occurrence of autoimmunity, by immunological aberrations due to the adjuvant-like effect of silicosis on the immunocyte system. The association of latent autoimmune hemolytic syndrome with pulmonary silicosis associated with tuberculosis seems likely to be induced by immunological aberration. The association of latent autoimmune hemolytic syndrome with Hodgkin's disease seems likely to be induced by the dysplasia of immunocyte system.

Kuroyanagi has reported the common antigenicity between normal human red blood cells and human leukemic cells. Anti-leukemic cell antibodies cross-react with normal leukocytes. It is well established that anti-leukocyte autoantibodies are frequently demonstrated in patients with leukemia. ${ }^{13-16}$ It is interesting that three leukemic patients demonstrated latent autoimmune hemolytic syndrome. These facts suggest that the positive direct antiglobulin test in these leukemic patients might be caused probably by the sensitization of their red blood cells with anti-leukemic cell autoantibodies.

It remains to be determined in future whether patients associated with latent autoimmune hemolytic anemia might develop manifest signs and symptoms of autoimmune hemolytic anemia.

\section{References}

1) Hennemann, H.H. Antikörperbedingte okkulte hämolytische Syndrome. Verh. dtsch. Ges. inn. Med., 1957, 63, 682-686.

2) Hennemann, H.H. Okkulte (latente) erworbene hämolytische Syndrome. Dtsch. med. Wschr., 1963, 88, 2288-2293.

3) Gajdusek, D.C. An 'auto-immune' reaction against human tissue antigen in certain chronic diseases. Nature, 1957, 179, 666-668.

4) Shulman, R., Marder, V.J., Hiller, M.C. \& Collier, E.M. Platelet and leukocyte 
isoantigens and their antibodies serologic, physiologic and clinical studies. Progress in Hematology, Vol. IV. 1964, pp. 222-304.

5) Payne, R. Leukocyte agglutinins in human sera. Arch. intern. Med., 1957, 99, 587606.

6) Dausset, J., Colombani, J. \& Okochi, K. Leukocyte and platelet agglutination. In: Immunological Method, Blackwell, Oxford, 1964, pp. 539-573.

7) Fukoka, Y. Clinical study on immunohemolytic anemia. Proceedings of the 17th General Assembly of the Japan Medical Congress, Vol. 2, 1967, pp. 336-342.

8) Kuroyanagi, T., Takahashi, R., Uono, K. \& Saito, M. Autoimmunity and thymus in patients with myasthenia gravis (In Japanese). Jap. J. clin. Path., 1967, 15, 472482.

9) Amano, S., Unno, G. \& Hanaoka, M. Studies on the differentiation of lymphocytes and plasma cells. An advocation of lymphogonia theory. Acta haemat. jap., 1951, 14, $108-114$.

10) Dameshek, W. 'Immunoblasts' and 'immunocytes' - an attempt at a functional nomenclature. Blood, 1963, 21, 243-245.

11) Kamijima, K. Silicosis as an adjuvant disease of man. Shikoku Igaku, 1968, 24, 513528 .

12) Kuroyanagi, T. \& Saito, M. Intracellular localization of antihuman leukemic cell antibodies and their effect on the enzyme activity of leukemic cells. Abstract of XII. Congress of Internal. Soc. Hematology, New York, 1968.

13) Tullis, J.L. Prevalence, nature and identification of leukocyte antibodies. New Eng. J. Med., 1958, 258, 569-578.

14) Tullis, J.L. Recent advance in the isolation and clinical significance of the antileukocytic protein from normal and pathologic sera. Proceedings of VIII. Internl. Congress of Hematology, 1960, pp. 1354-1359.

15) Walford, R.L. Leukocytes Antigens and Antibodies. Grune \& Stratton, New York, 1960.

16) Dameshek, W. \& Schwartz, R.S. Leukemia and auto-immumization-some possible relationships. Blood, 1959, 14, 1151-1158. 